\title{
Corrigendum
}

\section{Corrigendum to "A Case of Epistaxis as the First Sign of Acute Idiopathic Thrombocytopenic Purpura"}

\author{
Shori Tajima (D), Fumihiko Matsumoto, Takashi Anzai (D), Satoshi Hara, Yo Suzuki, \\ and Katsuhisa Ikeda
}

Department of Otorhinolaryngology, Juntendo University Faculty of Medicine, Tokyo 113-8421, Japan

Correspondence should be addressed to Shori Tajima; s-tajima@juntendo.ac.jp

Received 30 March 2021; Accepted 30 March 2021; Published 13 April 2021

Copyright (c) 2021 Shori Tajima et al. This is an open access article distributed under the Creative Commons Attribution License, which permits unrestricted use, distribution, and reproduction in any medium, provided the original work is properly cited.

In the article titled "A Case of Epistaxis as the First Sign of Acute Idiopathic Thrombocytopenic Purpura” [1], Figure 1 was formatted incorrectly. The authors have corrected this error and provided the correct Figure 1 as follows:

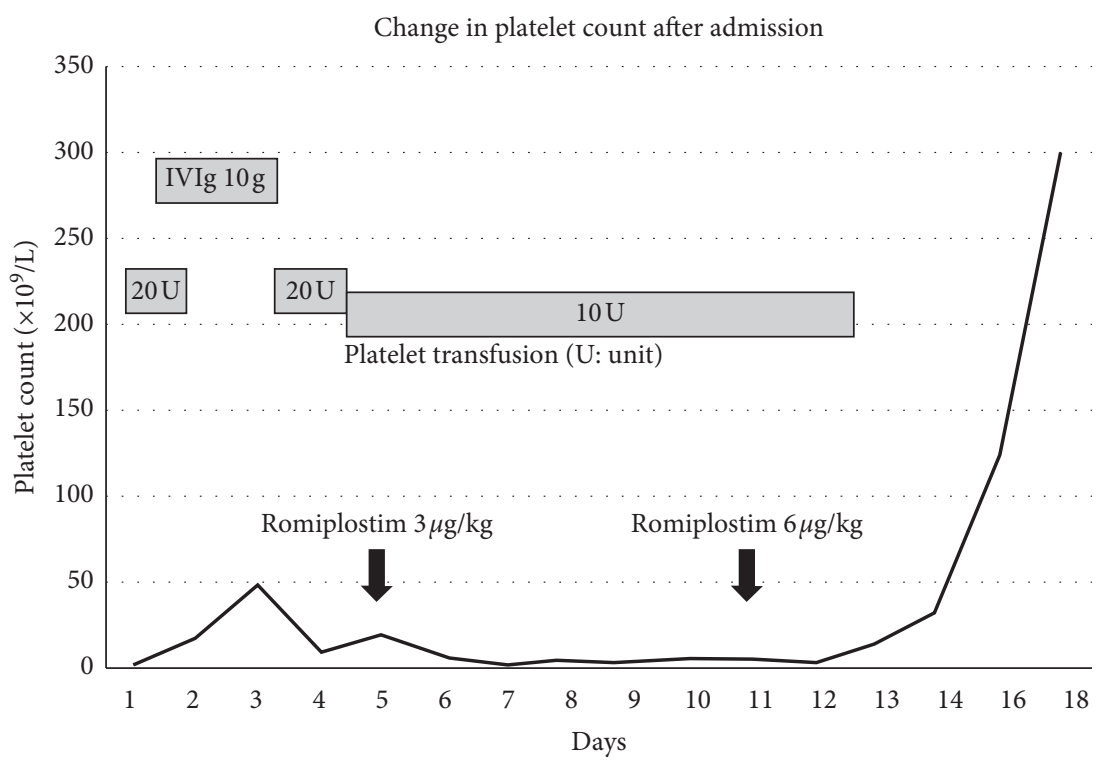

Figure 1: Change in platelet count after admission. The patient underwent treatment with platelet transfusion, high-dose intravenous gamma-globulin (IVIG), and romiplostim. Platelet counts increased about 14 days after admission. 


\section{References}

[1] S. Tajima, F. Matsumoto, T. Anzai, S. Hara, Yo Suzuki, and K. Ikeda, "A Case of Epistaxis as the First Sign of Acute Idiopathic Thrombocytopenic Purpura," Case Reports in Otolaryngology, vol. 2021, Article ID 6612939, 4 pages, 2021. 